\title{
Bubble-sort approach to channel routing
}

\author{
S.-S.Chen, C.-H.Yang and S.-J.Chen
}

\begin{abstract}
An efficient bubble-sort technique for solving the two-layer non-Manhattan channelrouting problem is presented. The time and space complexities of our algorithm are $\mathrm{O}(\mathrm{kn})$ and $\mathrm{O}(n)$, respectively, where $k$ is the number of sorting passes required and $n$ is the total number of two-terminal nets in a routing channel. The algorithm is easily extended to handle the cases with multiterminal nets distributed in a channel. Various tests verify the efficiency of the bubble-sort based router. Experimental results indicate that the router is time-efficient for routing. A threelayer algorithm having $\mathrm{O}(k n)$ time based on an identical problem formulation is proposed for solving the non-Manhattan channel routing.
\end{abstract}

\section{Introduction}

Channel routing plays an important role in minimising the routing area at the physical design level of VLSI circuits. In the last two decades various channel routing results have been reported based on the Manhattan model [1-7], which restricts the routing wires to be either vertical or horizontal. An example of the Manhattan routing is shown in Fig. 1, which solution needs some extra columns. Nowadays, the VLSI fabrication process does not preclude a layout style to be in a non-Manhattan routing model. As shown in Fig. 2 , the vertical constraints which occurred in the Manhattan routing model no longer exist in the non-Manhattan case.

The first non-Manhattan diagonal channel router, introduced by Lodi, et al. [8], realises a layout of two-terminal nets on a two-layer channel using a diagonal channel routing model (DCRM), where only wires of $45^{\circ}$ and $-45^{\circ}$ can be used. Later, Wang and Kuh $[9,10]$ proposed a mini-swap sorting method to solve the non-Manhattan channel routing problem. Fig. 3 shows a four-track solution for the example of Fig. 1 using Wang's mini-swap router.

A heuristic algorithm for the non-Manhattan channel routing based on the bubble-sort technique has been proposed by Chaudhary and Robinson [11]. The basic concept of the algorithm was derived from the inversion table introduced by Knuth [12]. Two types of inversion tables were used in Chaudhary's algorithm: left and right inversion tables. The criteria of sorting direction depends on the numbers of nonzero elements in the left and the right inversion tables. The sorting direction is from left to right (right-step) if the number of nonzero entries in the left inversion table is greater than that in the right inversion table and vice versa. But the algorithm hides a shortcoming, the sorting direction is set as from right to left

\section{(C) IEE, 2000}

IEE Proceedings online no. 20000810

DOI: $10.1049 /$ ip-cdt:20000810

Paper first received 1st July 1999 and in revised form 28th July 2000

S.-S. Chen and S.-J. Chen are with the Department of Electrical Engineering, National Taiwan University, Taipei, Taiwan, Republic of China

C.-H. Yang is with the Department of Information Management, Kun Shan University of Technology, Tainan, Taiwan, Republic of China

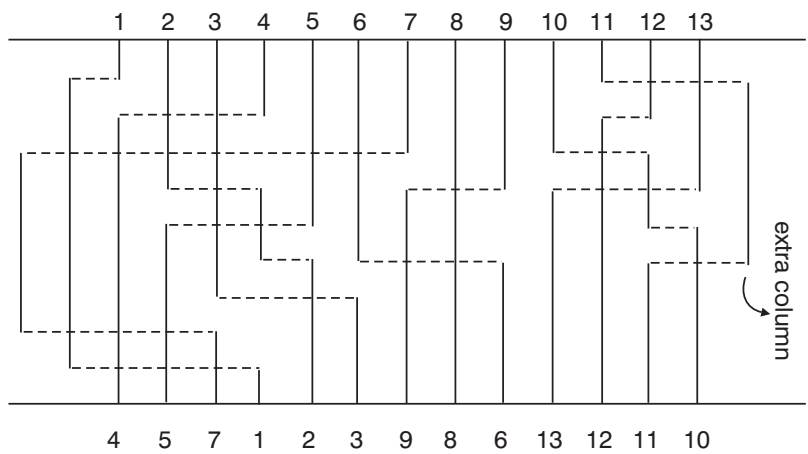

Fig. 1 Nine-track channel routing using Manhattan model

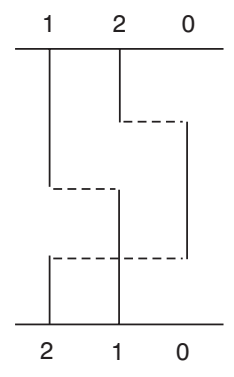

a

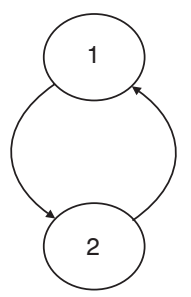

$b$

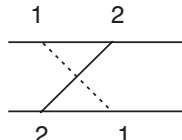

Fig. 2 Vertical constraints to not list in non-manhattan model a Manhattan model

$b$ Cyclic constraint graph

$c$ Diagonal model 


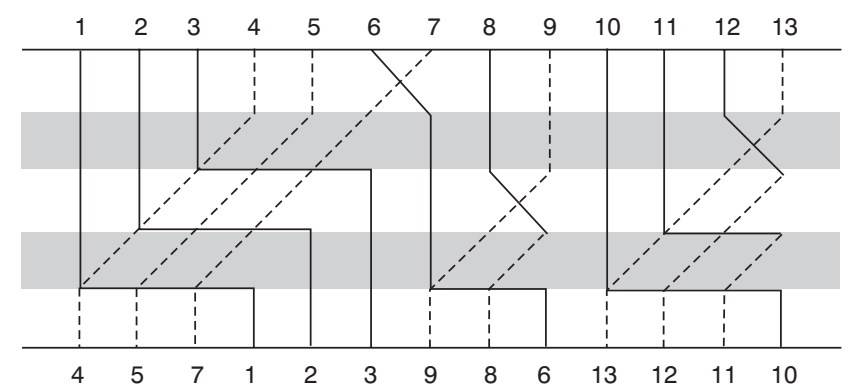

Fig. 4 Four-track solution for Fig. 1 using Chaudhary's routing model

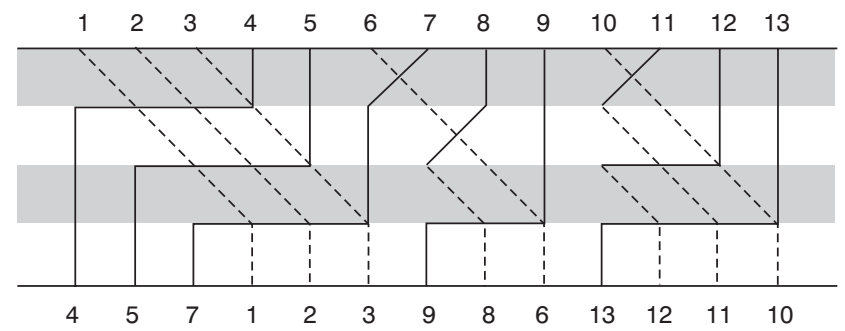

Fig. 5 Optimal three-track solution is obtained using our approach

(left-step) when the numbers of nonzero entries in the left and in the right inversion tables are equal. Therefore it cannot guarantee an optimal solution in terms of routing tracks required. Intuitively, the example shown in Fig. 4 is nonoptimal because a truly optimal solution needs only three routing tracks. In addition, the time and space complexities of the algorithm are $\mathrm{O}\left(k n^{2}\right)$ and $\mathrm{O}(n)$, respectively, where $k$ is the number of sorting passes required and $n$ is the total number of two-terminal nets in a routing channel.

Recently, an optimal bubble-sort algorithm in terms of routing tracks for the non-Manhattan channel routing has been proposed by Chen, et al. [13]. The optimal sorting sequence is generated by applying a left-step sorting and a right-step sorting to the nonequivalent sorting vectors in each pass of the bubble-sort. The procedure is iterated until some completely sorted vectors are found. This kind of enumerative algorithm can achieve an optimal solution with minimum number of sorting passes required. But the time and space complexities of the algorithm are not optimal because they still preserve an order of $\mathrm{O}\left(k^{2} n\right)$ and $\mathrm{O}(k n)$, respectively. We present in this paper a bubble-sort based algorithm under the same problem formulation as in $[9,11,13]$ and this algorithm can be extended easily to handle the cases of multiterminal nets. The time and space complexities of our algorithm are $\mathrm{O}(k n)$ and $\mathrm{O}(n)$, respectively. Obviously, our algorithm has a significant improvement in time and space complexities over the existing algorithms [11, 13]. An optimal routing solution for the example in Fig. 1 using our algorithm is shown in Fig. 5.

\section{Problem formulation}

Our research on non-Manhattan channel routing carries on with previous literature, such as Wang [9], Chaudhary [11], and Chen [13]. A non-Manhattan router has the following advantages over its Manhattan counterpart: The number of routing tracks and vias required is less than the Manhattan router (see Fig. 8 in Wang [9] and Fig. 1 in Chen [13]); the vertical constraints are not necessary; the total wire length is shorter than the Manhattan router; and no need of using

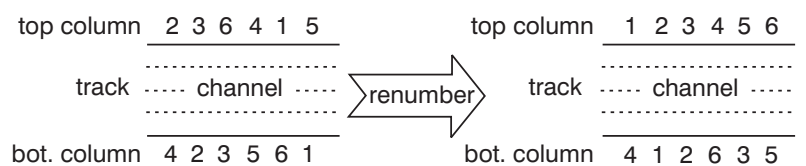

a

$b$

Fig. 6 Two identical channel routing problems

$a$ Original channel

$b$ Renumbered channel

extra columns outside the width of a channel to have the routing task done. For simplicity and making the concept clear, we assume that each net has two terminals over a channel, one terminal is from the top column vector and the other from the bottom column vector. The handling of multiterminal nets is described later. At first the terminals located in these column vectors are randomly labelled as integers. But we renumber the terminal labels in the top column vector to be 1 through $n$ and corresponding changes are made to the bottom terminal labels. For instance, assume that the top column vector $=(2,3,6,4$, $1,5)$ and the bottom column vector $=(4,2,3,5,6,1)$. If the top column vector is renumbered as $(1,2,3,4,5,6)$, the bottom column vector will be changed to $(4,1,2,6$, $3,5)$, as shown in Fig. 6.

The objective of channel routing is to interconnect nets having the same terminal labels and to minimise the number of routing tracks required, the time, and the space complexities as much as possible. In this paper we apply the bubble-sort technique to accomplish channel routing. Before describing our algorithm for the nonManhattan channel routing, we first define some terminologies as follows.

Definition 1: Left major table. $\left(b_{1}, b_{2}, \ldots, b_{n}\right)$ is said to be the left major table of a permutation $V=\left(v_{1}, v_{2}, \ldots, v_{n}\right)$ if $b_{v_{j}}$ is set to 1 , when there exists in $V$ at least one element to the left of $v_{j}$ and greater than $v_{j}$, for $j=1$ to $n$ and $v_{j} \in V$; otherwise, $b_{v_{i}}$ is set to 0 .

For example, given a permutation $V=(5,1,9,2,8,6,4$, $7,3)$, we have the following left major table $(1,1,1,1,0$, $1,1,1,0)$.

Definition 2: Right minor table. $\left(c_{1}, c_{2}, \ldots, c_{n}\right)$ is said to be the right minor table of a permutation $V=\left(v_{1}\right.$, $\left.v_{2}, \ldots, v_{n}\right)$ if $c_{v_{j}}$ is set to 1 , when there exists in $V$ at least one element to the right of $v_{j}$ and less than $v_{j}$, for $j=n$ to 1 and $v_{j} \in V$; otherwise, $c_{v_{j}}$ is set to 0 .

For example, given the permutation $V=(5,1,9,2,8,6$, $4,7,3)$ we have the following right minor table $(0,0,0,1$, $1,1,1,1,1)$.

Definition 3: Lexicographical ordering. Given two tables $B=\left(b_{1}, b_{2}, \ldots, b_{n}\right)$ and $C=\left(c_{1}, c_{2}, \ldots, c_{n}\right)$ which belong to a left major table and a right minor table or vice versa. Their lexicographical order is determined as follows:

(i) Initially, let $j$ be equal to 1 .

(ii) If $b_{j}>c_{j}\left(\right.$ or $c_{j}>b_{j}$ ) we say that $B$ is greater (less) than $C$ and can immediately terminate the comparison between $B$ and $C$.

(iii) Otherwise, if $b_{j}=c_{j}$ we increase the index $j$ by 1 and continue steps 2 and 3 to compare the next pair of elements in the tables until $j=n$. Besides, we say $B=C$ if $b_{j}=c_{j}$ for all $j=1$ to $n$.

For instance, given two tables $B=(1,0,1,1,0,1,1,1,0)$ and $C=(1,0,1,0,0,1,1,1,1)$, we say that table $B$ is greater than table $C$.

IEE Proc.-Comput. Digit. Tech, Vol. 147, No. 6, November 2000 


\section{Two-layer non-Manhattan channel routing}

\subsection{Non-Manhattan routing model}

Traditionally, the most common channel routing model, known as a Manhattan model, uses vertical and horizontal wires for routing (also known as an $\mathrm{H}-\mathrm{V}$ routing model) on two or more layers over a channel. In a non-Manhattan channel routing model three wiring types are used: diagonal (run at $45^{\circ}$ or $-45^{\circ}$ direction), horizontal and vertical wires as shown in Fig. 7. The routing model we use is a non-Manhattan model with two available layers for the routing of two-terminal nets over a channel. We reserve one layer for metal-1 wires and the other layer for metal-2 wires and a contact cut is required while making connection between these two layers.

Chaudhary [11] made an interesting observation about the bubble-sort as follows. Given a vector $V=$ $\left(v_{1}, v_{2}, \ldots, v_{n}\right)$, if the sorting direction for $V$ either traversing from left to right (denoted as right-step) or from right to left (denoted as left-step) in each bubble sorting pass is fixed, it will yield worse results in terms of total number of routing tracks in a channel. Intuitively, one can obtain an optimal result if the sorting direction has been properly determined such that elements in $V$ can be moved more steps (distances) in each pass of the bubble-sort. The effects of using different types of sorting directions on the final routing tracks are shown in Fig. 7. Therefore we have to further explain in the following Section how to determine the sorting direction by using the left major and the right minor tables in each of the sorting passes.

\subsection{Algorithm for bubble-sort router}

We propose an efficient bubble-sort algorithm for the nonManhattan channel routing. First, assume that the unsorted and bottom column-vector is the input sorting vector. The algorithm has two important phases. The purpose of first phase is to generate both the left major table and the right minor table for a sorting vector. Let the sorting vector $V=\left(v_{1}, v_{2}, \ldots, v_{n}\right)$ be a permutation of the natural sequence $(1,2, \ldots, n)$. Entries in the left major table and the right minor table are generated by applying the following lemma.

Lemma 1: For a sorting vector $V=\left(v_{1}, v_{2}, \ldots, v_{n}\right)$, the left major table and the right minor table of $V$ in each of sorting passes can be constructed in $T_{a}(n)=\mathrm{O}(n)$ time each, where $n$ is the number of elements in $V$.

Proof: Let $\left(b_{1}, b_{2}, \ldots, b_{n}\right)$ and $\left(c_{1}, c_{2}, \ldots, c_{n}\right)$ be the left major table and the right minor table of this vector $V$, respectively. These two tables are constructed as follows. If $v_{j}>$ Max $\_a l$, then set $b_{v_{j}}$ to a value of zero and replace Max $\_$val with $v_{j}$. Otherwise set $b_{v}$ to a value of one; for $j=1,2, \ldots, n$ (from left to right). Obviously, the left major table construction takes $\mathrm{O}(n)$ time. Similarly, if

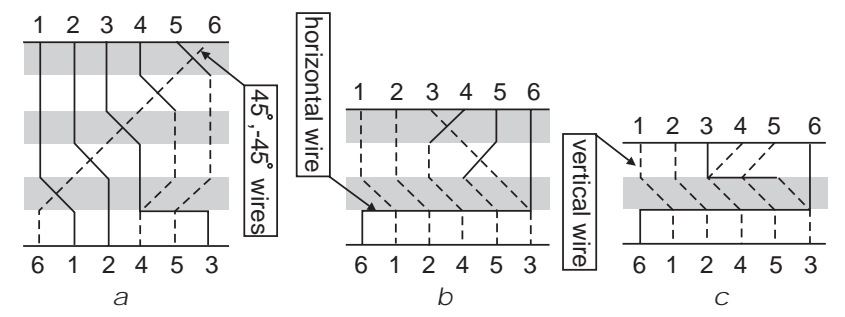

Fig. 7 Effects of different sorting directions

a Left-step only

$b$ Right-step only

c Left- \& right-steps. $v_{j}<$ Min $\_$val, then set $c_{v_{j}}$ to a value of zero and replace Min_val with $v_{j}$. Otherwise set $c_{v_{j}}$ to a value of one; for $j=n, n-1, \ldots, 2,1$ (from right to left). This right minor table construction also takes $\mathrm{O}(n)$ time. Here the initial values of $M a x_{-}$val and Min_val are zero and a large positive number, respectively.

The second phase purpose is to properly choose a sorting direction for each pass of the bubble-sort, since a good sorting direction will reduce the number of sorting passes required by the bubble-sort. From the previous phase we have constructed two sets of tables: the left major table and the right minor table. We choose the sorting direction to be left-step (respectively, right-step) if the number of nonzero entries in the left major table is less (respectively, greater) than that in the right minor table. If the numbers of nonzero entries in the left major table and in the right minor table are equal, we must compare their lexicographical order. We choose the sorting direction to be right-step if the left major table is greater than the right minor table referring to the lexicographical ordering definition in Section 2; and left-step otherwise. Once the sorting direction for the sorting vector is determined a pass of bubble-sort is performed. These phases are repeated until the completely sorted vector is found.

To make the principle of our algorithm clear we describe the sorting passes of a non-Manhattan channel routing as follows. Given an example with a top column vector $=$ $(1,2,3,4,5,6,7,8,9)$ and a bottom column vector $=$ $(2,3,9,4,5,6,7,8,1)$. The bubble sorting process begins with the bottom sorting vector. At each intermediate step of the sorting, a new sorting vector is generated as the result of a permutation of the natural sequence $(1,2, \ldots, n)$ and this vector will require one routing track in a channel. Initially, the left major table and the right minor table can be constructed by scanning the input sorting vector. For this example, we have the first left major table and the first right minor table as $(1,0,0,1,1,1,1,1,0)$ and $(0,1,1,1$, $1,1,1,1,1)$, respectively. Obviously, the numbers of nonzero entries in the left major table and the right minor table are 6 and 8, respectively. Hence the sorting direction will be chosen as left-step. After having performed this left-step bubble-sort, a new sorting vector is produced as $(1,2,3,9,4,5,6,7,8)$. Similarly, in the second pass, the left major table and the right minor table constructed are $(0,0,0,1,1,1,1,1,0)$ and $(0,0,0,0,0,0$, $0,0,1)$, respectively. As the numbers of nonzero entries in the left major table and the right minor table are 5 and 1 , respectively, the sorting direction will be chosen as rightstep and the current sorting vector is changed to $(1,2,3,4$, $5,6,7,8,9)$ which is already in order. Therefore two passes of bubble-sort are required to complete the sorting work. The illustration for the example is shown in Fig. 8 and its corresponding non-Manhattan channel routing solution is plotted in Fig. 9. The description of our bubble-sort router is depicted in algorithm 1.

Theorem 1: The time and space complexities of using our bubble-sort algorithm to route a two-layer non-Manhattan channel take $T_{b}(n)=\mathrm{O}(k n)$ time and $\mathrm{O}(n)$ space, respectively, where $k$ is the number of sorting passes required and $n$ is the number of two-terminal nets in a channel.

Proof: In each of the bubble-sorting passes the sorting task for a sorting vector $V=\left(v_{1}, v_{2}, \ldots, v_{n}\right)$ will be performed by choosing a left-step or a right-step direction, which runs in $\mathrm{O}(n)$ time. Referring to lemma 1 , we need $T_{a}(n)=\mathrm{O}(n)$ time to generate a table in each sorting pass. Assume that the sorting task is completely sorted after the $k$ th bubble- 


\begin{tabular}{|c|c|c|c|c|}
\hline \multirow{2}{*}{$\begin{array}{l}\text { Pass } \\
\text { no. }\end{array}$} & Sorting vector & \multirow[t]{2}{*}{$M a x_{-} v a l$} & \multirow{2}{*}{$\frac{\text { Sorting vector }}{\text { Right minor table }}$} & \multirow[t]{2}{*}{ Min_val } \\
\hline & Left major table & & & \\
\hline \multirow[t]{13}{*}{1} & (1) (2)(3)(4)(6)(1) 8(9) & \multirow[t]{3}{*}{2} & (1) (2)(3) (4)(5) (6) (7) 89) & \multirow[t]{3}{*}{1} \\
\hline & $(239456781)$ & & $(239456781)$ & \\
\hline & $(\square 0 \square \square \square \square \square)$ & & $(0 \square \square \square \square \square \square()$ & \\
\hline & $(239456781)$ & \multirow[t]{2}{*}{3} & $(239456781)$ & \multirow[t]{2}{*}{1} \\
\hline & 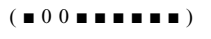 & & $(0 \sqcap \square \square \square=1 \backsim)$ & \\
\hline & $(239456781)$ & \multirow[t]{2}{*}{9} & $(239456781)$ & \multirow[t]{2}{*}{1} \\
\hline & $\left(\begin{array}{lll}0 & 0 & \square \\
\end{array}\right.$ & & 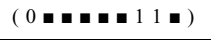 & \\
\hline & & \multicolumn{2}{|c|}{ the operation continues... } & \\
\hline & $(239456781)$ & \multirow[t]{2}{*}{9} & $(239456781)$ & \multirow[t]{2}{*}{1} \\
\hline & 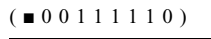 & & $(0 \quad 011111111111)$ & \\
\hline & $(239456781)$ & \multirow[t]{2}{*}{9} & $(239456781)$ & \multirow[t]{2}{*}{1} \\
\hline & $\left(\begin{array}{lllllllll}1 & 0 & 0 & 1 & 1 & 1 & 1 & 1 & 0\end{array}\right)$ & & $\left(\begin{array}{lllllllll}0 & 1 & 1 & 1 & 1 & 1 & 1 & 1 & 1\end{array}\right)$ & \\
\hline & $\begin{array}{l}\text { New sorting vector (1 } 2 \\
\text { bubble-sort to current sc }\end{array}$ & $\begin{array}{l}45678) \text { i } \\
\text { vector. }\end{array}$ & erated by applying a pass & eft-step \\
\hline \multirow[t]{7}{*}{2} & $(123945678)$ & \multirow[t]{2}{*}{1} & $(123945678)$ & \multirow[t]{2}{*}{8} \\
\hline & 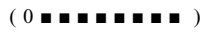 & & 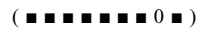 & \\
\hline & & \multicolumn{2}{|c|}{ the operation continues... } & \\
\hline & $(123945678)$ & \multirow[t]{2}{*}{9} & $(123945678)$ & \multirow[t]{2}{*}{2} \\
\hline & $\left(\begin{array}{llllllll}0 & 0 & 0 & 1 & 1 & 1 & 1 & \square\end{array}\right)$ & & 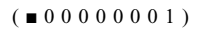 & \\
\hline & $(123945678)$ & \multirow[t]{2}{*}{9} & $(123945678)$ & \multirow[t]{2}{*}{1} \\
\hline & $\left(\begin{array}{lllllllll}0 & 0 & 0 & 1 & 1 & 1 & 1 & 1 & 0\end{array}\right)$ & & 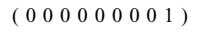 & \\
\hline
\end{tabular}

Fig. 8 Example to illustrate sorting process of our algorithm

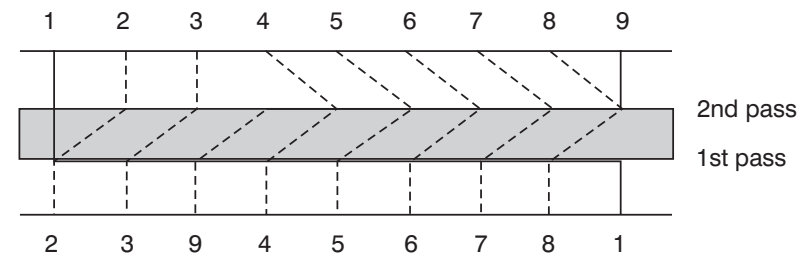

Fig. 9 Non-Manhattan channel routing solution corresponding to Fig. 8

sorting pass. As a result the overall time complexity $T_{b}(n)$ of our algorithm is formulated as

$$
\begin{aligned}
T_{b}(n) & =\sum_{i=1}^{k}\left[n+2 T_{a}(n)\right]=\sum_{i=1}^{k}[n+2 n] \\
& =3 \sum_{i=1}^{k} n=3 k n \cong O(k n)
\end{aligned}
$$

In the worst case the time complexity takes $\mathrm{O}\left(n^{2}\right)$ when all elements in a sorting vector $V$ are in reverse order and then $k$ is asymptotic to $n$. On the space complexity side, for storing the information needed in sorting vectors, the left major table and the right minor table used in all of the sorting passes need $n$ space each. Obviously, the total space complexity of our algorithm is preserved in $\mathrm{O}(n)$ because the required space for the sorting vectors and tables is reusable in each sorting pass of the bubble-sort.

\subsection{Handling of multiple terminal nets}

In general, multiterminal nets usually exist in a routing channel. We first transform these multiterminal nets into sets of two-terminal nets before the bubble-sort router. For simplicity, we classify these multiterminal nets into type-I,

\section{Algorithm 1: Two-layer bubble-sort channel router}

Algorithm TwoLayer_BubbleSortRouter()

\{

$P A S S=0$

Sorting_Vector $=$ Input_Vector ;

write( Sorting_Vector);

while( unsorted for Sorting_Vector ) \{ Compute_Tables( Sorting_Vector ); Set_Step_Type( Sorting_Direction ); switch( Sorting_Direction ) \{

case L_to_R : / ${ }^{*}$ the sorting direction is right-step ${ }^{*} /$ for $(\mathrm{j}=1 ; \mathrm{j}<\mathrm{n} ; \mathrm{j}++)$

if ( Sorting_Vector[j] > Sorting_Vector $[j+1]$ ) break; swap( Sorting_Vector[j], Sorting_Vector[j +1$]$ )

case R_to_L : /* the sorting direction is left-step $* /$ for $(j=n ; j>1 ; j--)$

if ( Sorting_Vector[j] $<$ Sorting_Vector $[j-1]$ ) swap( Sorting_Vector[j], Sorting_Vector $[\mathrm{j}-1]$ );

write( Sorting_Vector ); $/{ }^{*}$ output the sorting vector to a file */

PASS ++; $/{ }^{*}$ increase the number of sorting passes by one $^{*} /$ \}

\}$/^{*}$ end of the TwoLayer_BubbleSortRouter() Algorithm */

Compute_Tables( Sorting_Vector)

$\left\{/^{*}\right.$ compute the left major table and the right minor table* $/$

Initialize Left_table, Right_table;

Left_val $=$ Right_val $=0$;

Max_val $=0$;

for $(\mathrm{j}=1 ; \mathrm{j}<=\mathrm{n} ; \mathrm{j}++) /{ }^{*}$ construct the left major table ${ }^{*} /$

if ( Sorting_Vector[j] > Max_val)

Left_table[ Sorting_Vector[j]] $=0$;

Max_val = Sorting_Vector[j];

else

Left_table[ Sorting_Vector[j]] = ;

Left_val ++;

Min_val $=\infty$

for $(\mathrm{j}=\mathrm{n} ; \mathrm{j}>=1 ; j--) /{ }^{*}$ construct the right minor table $* /$

if ( Sorting_Vector[j] < Min_val)

Right_table[ Sorting_Vector[j]] $=0$;

Min_val $=$ Sorting_Vector[j]

else

Right_table[ Sorting_Vector[j]] $=1$;

Right_val ++;

\} /* end of the subroutine */

Set_Step_Type(Sorting_Direction)

$\left\{/{ }^{*}\right.$ decide the sorting direction for a sorting vector *

switch(Left_val - Right_val) \{

case $>0$ : Sorting_Direction $=$ L_to_R ; break;

case $=0$ : if (Left_table $>$ Right_table in terms of lexicographical order $)$ Sorting_Direction $=$ L_to_R;

else Sorting_Direction $=$ R_to_L;

break;

case $<0$ : Sorting_Direction $=$ R_to_L;

\}

\}/ end of the subroutine */

type-II and type-III nets. A net is called type-I net if their terminals are distributed only on the upper or lower side of a channel. For example, the terminals $\left(b_{1}, b_{2}\right)$ of net $b$ in Fig. $10 a$ can be directly routed together and be dropped from consideration in later sorting phase. A type-II $m$-terminals net has $m$-1 terminals $(m \geq 2)$ on one side and only one terminal on the opposite side of a channel. If the $m-1$ terminals are on the upper side, we first connect them together and then one of the terminals on this side and the only terminal on the lower side are chosen to form a two-terminal net to be routed in a later sorting phase. 

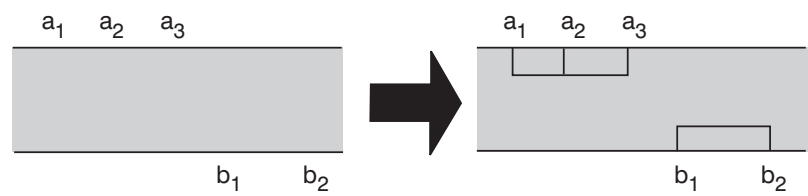

a
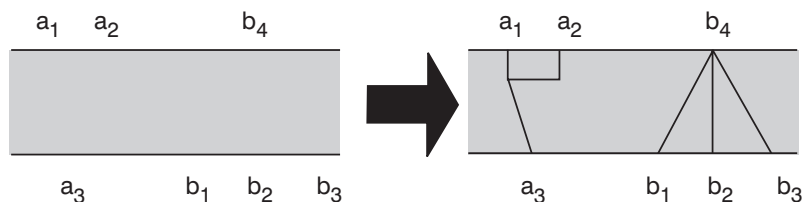

$\begin{array}{llll}a_{3} & b_{1} & b_{2} & b_{3}\end{array}$

b
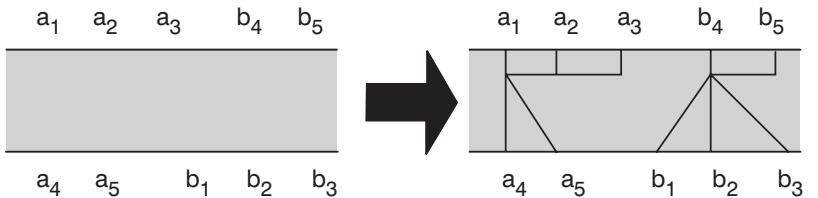

Fig. 10 Classification of multiterminal nets in bubble-sort router $a$ Type-I nets

$b$ Type-II nets

$c$ Type-III nets

Similarly, if the $m-1$ terminals are located on the lower side, they will be connected to the upper terminal at a single column in later sorting phase. This type-II case is plotted in Fig. 10b. A net is called type-III net if there are some $x$ terminals distributed on the upper side and some $(m-x)$ terminals on the lower side of a channel. This case is illustrated in Fig. $10 c$. For the upper $x$ terminals, we first connect them together and choose a suitable terminal as the final representative terminal. Then this upper representative terminal is connected to all $(m-x)$ terminals on the lower side as in the type-II case. After this process, all multiterminal nets can be transformed into two-terminal nets, and we can then apply the same bubble-sort router to complete the routing of multiterminal nets.

\section{Three-layer non-Manhattan channel routing}

Advances in VLSI technology mean that more than two layers are available for routing. We now extend the bubblesort router to solve the same routing problem over a threelayer channel.

During three-layer routing, our two-layer bubble-sort router (algorithm 1) can first be invoked to generate a sorting sequence $S$ for an unsorted vector $V=\left(v_{1}\right.$, $\left.v_{2}, \ldots, v_{n}\right)$. Each element in the sorting sequence is either a left-step $(L)$ or a right-step $(R)$. The sorted vector can be represented as $S V=A_{k} A_{k-1} \ldots A_{2} A_{1} V$, where $A_{i}$ is either a left-step pass or a right-step pass of bubble-sort and $1 \leq i \leq k$. Now we scan the $S$ from right to left until a pass of left-step bubble-sort, $A_{j}$, is found with index $j$ in $S$. In other words,

$$
S=A_{k} A_{k-1} \ldots A_{j+1} L \underbrace{R R \ldots R R}_{j-1} .
$$

Again, by repeatedly applying the theorem $L R V=R L V$ in [13], we have

$$
\begin{aligned}
S V & =A_{k} A_{k-1} \ldots A_{j+1} L \underbrace{R R \ldots R R}_{j-1} V=\ldots \\
& =A_{k} A_{k-1} \ldots A_{j+1} R \underbrace{R \ldots R R L}_{j-1} V .
\end{aligned}
$$

This process is iterated for the finding of left-steps until all left-step sorting passes in $S$ are moved to the right side of the sorting sequence $S$. Finally, the sorting sequence is represented as follows:

$$
\begin{aligned}
S V & =A_{k} L \underbrace{R R \ldots R}_{c-1} L L \ldots L V \\
& =\underbrace{R R R \ldots R}_{c} \underbrace{L L L \ldots L}_{k-c} V=R^{c} L^{k-c} V
\end{aligned}
$$

where $k$ is the number of sorting passes required for $V$ and $c$ is a constant, $0 \leq c \leq k$.

Lemma 2: The completely sorted sequence $S=R^{c} L^{k-c}$ of a sorting vector $V$ generated by our bubble-sort router can be adjusted to be $R$ (right-step) and $L$ (left-step) alternatively in $T_{c}(n)=\mathrm{O}(k)$ time, where $k$ is the number of sorting passes required for $V$ and $0 \leq c \leq k$.

Proof: Consider a completely sorted sequence $S=R^{c} L^{k-c}$ of a sorting vector $V$. Using the theorem $L R V=R L V$ as proved in [13], the sorting sequences of $L R$ and $R L$ in a bubble-sort solution to $V$ are interchangeable. That is, the sorting result only depends on the number of left-step $(L)$ or right-step $(R)$ passes rather than their permutation positions in a bubble-sort solution. As a result,

$$
S V=R^{c} L^{k-c} V=\left(L^{x} \mid R^{x}\right) \underbrace{R L R L \ldots R L} V
$$

is obtained in $\mathrm{O}(k)$ time, where $0 \leq x \leq k$. Here, the symbol $\left(L^{x} \mid R^{x}\right)$ represents the extra $L^{x}$ or $R^{x}$ when the numbers of $L s$ and $R s$ are not equal.

Since three layers are available for routing in a channel, if the routings of a pass of $R$ and a pass of $L$ bubble-sort occupy different horizontal layers, they can be integrated into a single track. Therefore the number of routing tracks for an unsorted vector $V$ is equal to the value of maximum $\{c, k-c\}$.

Following closely the foregoing discussion, the sorting result has been generated as a series of intermediate permutations. The criteria presented in [11] will be used for layer assignment of wire segments as follows. In an intermediate permutation the wire segments which have been moved toward to the right are assigned to layer 1, those moved toward the vertical are assigned to layer 2 , and those moved toward the left are assigned to layer 3 . This characteristic of layer assignment is similar to the conventional HVH Manhattan routing model. Finally, the routing is done by integrating a pair of adjacent intermediate permutations into one routing track in a channel. An outline of the three-layer routing algorithm is depicted in algorithm II. For example, given an unsorted vector

\section{Algorithm II: Three-layer bubble-sort channel router}

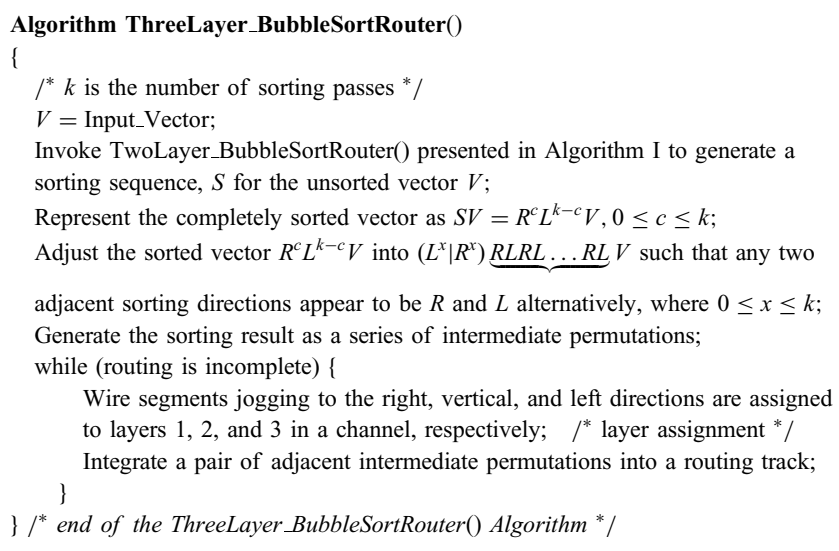




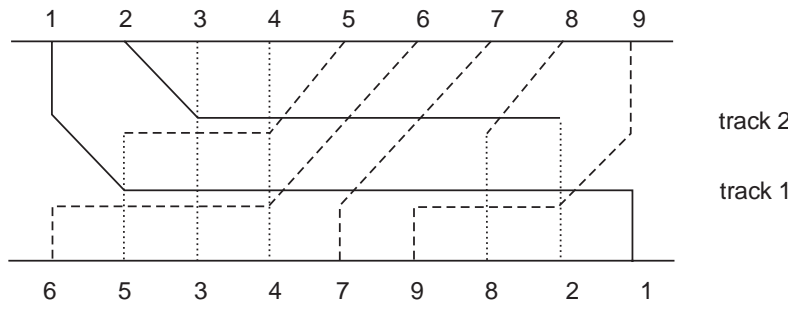

Fig. 11 Example of three-layer non-Manhattan channel routing solution using our bubble-sort router

$V=(6,5,3,4,7,9,8,2,1)$. A three-layer non-Manhattan channel routing solution $S V=R R L L V=R L R L V$ using our bubble-sort router is shown in Fig. 11.

Theorem 2: The time complexity $T_{d}(n)$ of using our bubble-sort algorithm to route a three-layer non-Manhattan channel takes $\mathrm{O}(k n)$ time, where $k$ and $n$ stand for the number of sorting passes required and the number of twoterminal nets in a channel, respectively.

Proof: The time complexity of our three-layer bubble-sort algorithm is calculated as follows. Consider a completely sorted vector $R^{c} L^{k-c} V$ obtained by our two-layer bubblesort based algorithm takes $T_{b}(n)$ time, where $V=\left(v_{1}, v_{2}, \ldots, v_{n}\right)$. By lemma 2 , to adjust the completely sorted sequence $R^{c} L^{k-c}$ such that any two adjacent sorting directions appear to be $R$ and $L$ alternatively takes $T_{c}(n)$ time. Formally stated, the overall time complexity $T_{d}(n)$ of our three-layer algorithm is

$$
T_{d}(n)=T_{b}(n)+T_{c}(n)=\mathrm{O}(k n)+\mathrm{O}(k) \cong \mathrm{O}(k n)
$$

As a result, the time complexity is bounded in $\mathrm{O}(\mathrm{kn})$ on the average. In the worst case, a time complexity $\mathrm{O}\left(n^{2}\right)$ is obtained when all elements in a sorting vector $V$ are in reverse order and $k$ is asymptotic to $n$.

\section{Experimental results}

Our bubble-sort channel router, Chaudhary's [11] router, and Chen's [13] router have been implemented in C language and tested on a Sun Ultra-SPARC-1 workstation running the Solaris 2.6 platform. Furthermore, we have conducted many testing examples collected from the literature $[9,11,13]$ and some cases originated by ourselves to evaluate the effectiveness and correctness of our router. The experimental results are compared and analysed as follows.

First, the results of running different routers on several benchmarks are shown in Table 1. From the testing results of Lodi [8] in Table 1, many situations of 'no solution' occur under the constraint of no allowance of using extra column(s) in a channel. Next, the comparisons of complexity among two-layer algorithms are listed in Table 2. In Table 2 the time complexity of the diagonal router proposed in [8] runs $\mathrm{O}(n)$. But it fails to complete the routing without magnifying the row spacing by $\sqrt{2}$ and without using extra column(s) in a channel for most of the cases. We next compare the experimental results with the mini-swap router [9] which has a time and a space complexities of $\mathrm{O}(k n)$ and $\mathrm{O}(n)$, respectively. But it could not generate optimal solutions in terms of routing tracks required. We also run the same instances using Chaud-

Table 1: Experimental results on running some benchmarks

\begin{tabular}{|c|c|c|c|c|c|c|c|}
\hline \multirow[b]{2}{*}{ Benchmarks } & \multicolumn{2}{|c|}{ Order of nets } & \multicolumn{3}{|c|}{ Routing tracks for different non-Manhattan routers } & \multirow[b]{2}{*}{ Chen [13] } & \multirow[b]{2}{*}{ ours } \\
\hline & terminals & nets & Lodi [8] & Wang [9] & Chaudhary [11] & & \\
\hline $\begin{array}{l}\text { Circuit } 1 \\
\text { Fig. } 8 \text { in [9] }\end{array}$ & 14 & 7 & $\begin{array}{l}\text { no } \\
\text { solution }\end{array}$ & 3 & 3 & 3 & 3 \\
\hline $\begin{array}{l}\text { Circuit } 2 \\
\text { Fig. } 3 \text { in [13] }\end{array}$ & 16 & 8 & $\begin{array}{l}\text { no } \\
\text { solution }\end{array}$ & 6 & 5 & 5 & 5 \\
\hline $\begin{array}{l}\text { Circuit } 3 \\
\text { Fig. } 7 \text { in [13] }\end{array}$ & 16 & 8 & 6 & 6 & 4 & 4 & 4 \\
\hline $\begin{array}{l}\text { Circuit } 4 \\
\text { Fig. } 5 \text { in [11] }\end{array}$ & 18 & 9 & $\begin{array}{l}\text { no } \\
\text { solution }\end{array}$ & 8 & 2 & 2 & 2 \\
\hline $\begin{array}{l}\text { Circuit } 5 \\
\text { Fig. } 5 \text { [our] }\end{array}$ & 26 & 13 & $\begin{array}{l}\text { no } \\
\text { solution }\end{array}$ & 4 & 4 & 3 & 3 \\
\hline $\begin{array}{l}\text { Circuit } 6 \\
\text { Fig. } 5 \text { in [13] }\end{array}$ & 32 & 16 & $\begin{array}{l}\text { no } \\
\text { solution }\end{array}$ & 5 & 5 & 3 & 3 \\
\hline $\begin{array}{l}\text { Circuit } 7 \\
\text { Fig. } 9 \text { in [9] }\end{array}$ & 34 & 17 & $\begin{array}{l}\text { no } \\
\text { solution }\end{array}$ & 7 & 5 & 5 & 5 \\
\hline
\end{tabular}

Table 2: Comparisons for two-layer routing algorithms

\begin{tabular}{llllll}
\hline \multicolumn{2}{c}{ Complexity } & \multicolumn{2}{l}{$\begin{array}{l}\text { Time complexity } \\
\text { average }\end{array}$} & worst & \multicolumn{2}{l}{$\begin{array}{l}\text { Space complexity } \\
\text { average }\end{array}$} & worst & $\begin{array}{l}\text { Bubble sort } \\
\text { approach? }\end{array}$ \\
\hline Lodi [8] & $\mathrm{O}(n)$ & no solution & need extra columns & NO \\
Wang [9] & $\mathrm{O}(k n)$ & $\mathrm{O}\left(n^{2}\right)$ & $\mathrm{O}(n)$ & $\mathrm{O}(n)$ & NO \\
Chaudhary [11] & $\mathrm{O}\left(k n^{2}\right)$ & $\mathrm{O}\left(n^{3}\right)$ & $\mathrm{O}(n)$ & $\mathrm{O}(n)$ & YES \\
Chen [13] & $\mathrm{O}\left(k^{2} n\right)$ & $\mathrm{O}\left(n^{3}\right)$ & $\mathrm{O}(k n)$ & $\mathrm{O}\left(n^{2}\right)$ & YES \\
Our Router & $\mathrm{O}(k n)$ & $\mathrm{O}\left(n^{2}\right)$ & $\mathrm{O}(n)$ & $\mathrm{O}(n)$ & YES \\
\hline
\end{tabular}


Table 3: Comparison with other routers for randomly generated examples

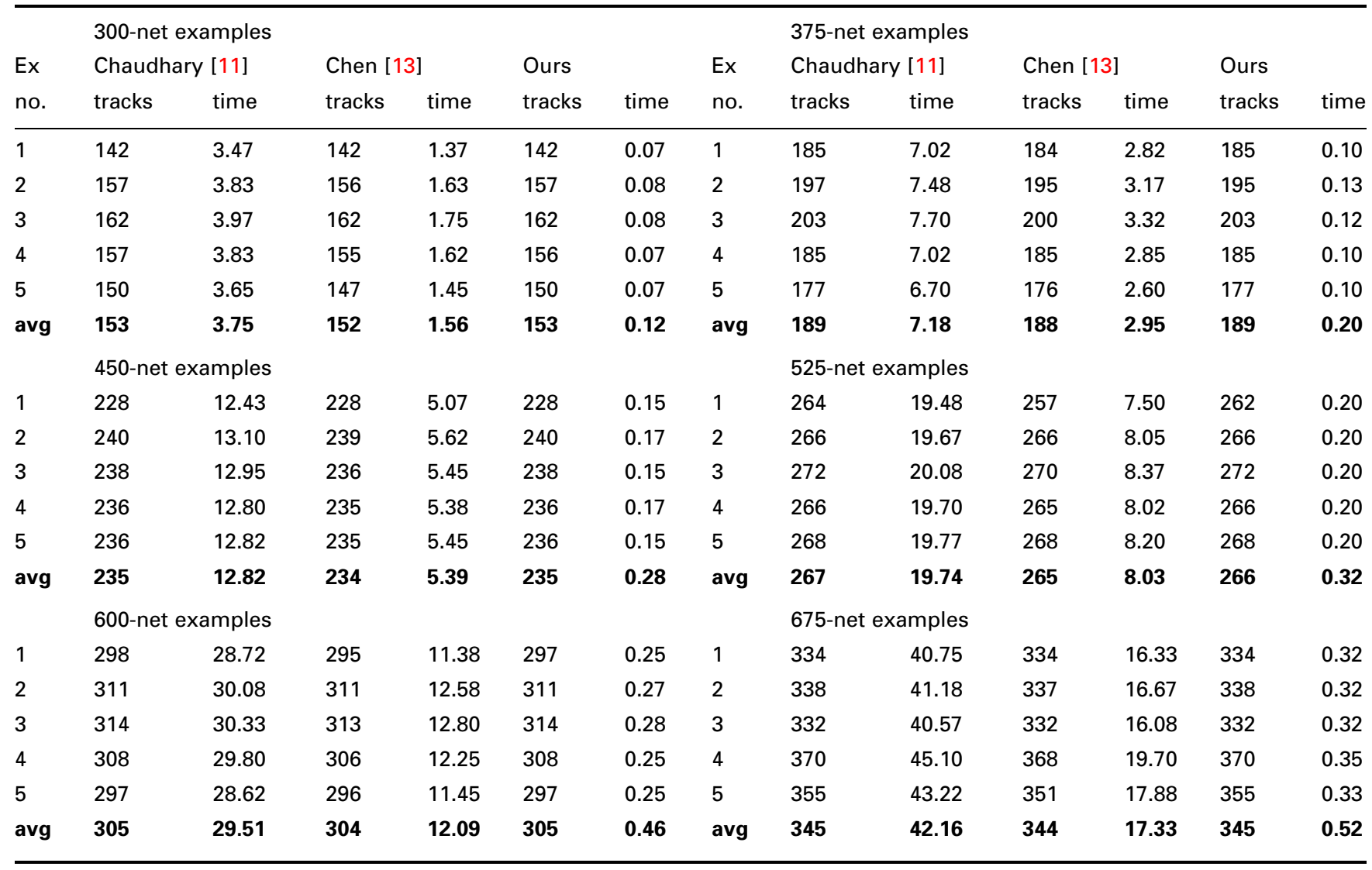

hary's [11] router. Its time complexity not only takes $\mathrm{O}\left(k n^{2}\right)$ on the average, even $\mathrm{O}\left(n^{3}\right)$ in the worst case (i.e. the case where all elements in a sorting vector are in reverse order). In general this router cannot generate an optimal solution in terms of routing tracks required and time performance. Chen [13] has presented an optimal router in terms of routing tracks required for the nonManhattan channel routing. But the time and space complexities of the router preserved a high order of $\mathrm{O}\left(k^{2} n\right)$ and $\mathrm{O}(k n)$ 'respectively' on the average, even $\mathrm{O}\left(n^{3}\right)$ time in the worst case, where $k$ is asymptotic to $n$. Our proposed algorithm spends $\mathrm{O}(k n)$ on the average or $\mathrm{O}\left(n^{2}\right)$ in the worst case for time complexity, and $\mathrm{O}(n)$ for space complexity.

To explore the features of our proposed router, we tested the last three bubble-sort based algorithms on randomly generated examples as shown in Table 3. Experimentally,

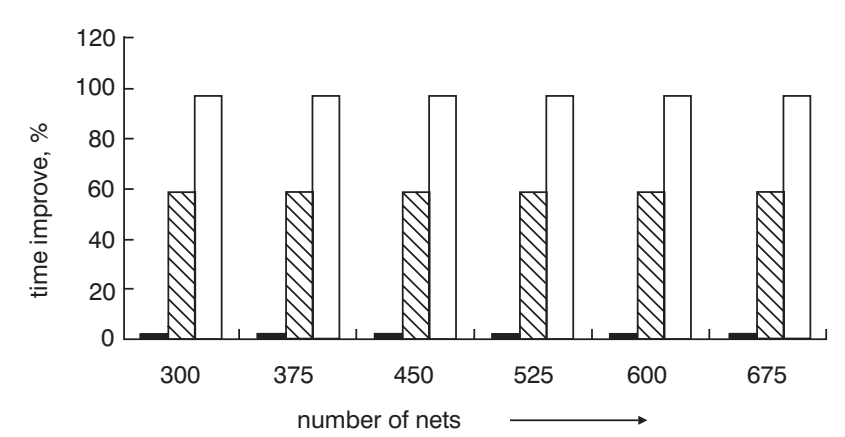

Fig. 12 Comparison on performance improvement for examples in Table 3

- Chaudhary [11]

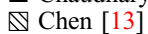

$\square$ Our router
Table 4: Comparisons for three-layer routing algorithms

\begin{tabular}{|c|c|c|c|c|}
\hline \multirow{2}{*}{ Complexity } & \multicolumn{2}{|c|}{ Time complexity } & \multicolumn{2}{|c|}{ Space complexity } \\
\hline & average & worst & average & worst \\
\hline Chaudhary [11] & $\mathrm{O}\left(k n^{2}\right)$ & $\mathrm{O}\left(n^{3}\right)$ & $\mathrm{O}(n)$ & $\mathrm{O}(n)$ \\
\hline Chen [13] & $\mathrm{O}\left(k^{2} n\right)$ & $\mathrm{O}\left(n^{3}\right)$ & $\mathrm{O}(k n)$ & $\mathrm{O}\left(n^{2}\right)$ \\
\hline Our router & $\mathrm{O}(k n)$ & $\mathrm{O}\left(n^{2}\right)$ & $\mathrm{O}(n)$ & $\mathrm{O}(n)$ \\
\hline
\end{tabular}

our CPU-time T (in seconds) performance is $97 \%$ better than that of [11] and $40 \%$ better than that of [13] as shown in Fig. 12. The time performance improvement of [11] is denoted as zero in Figure 12 because we compare both our and Chen's [13] routers to [11]. And the number of routing tracks required by our router as shown in Table 3 is only $1 \%$ more than that of the optimal router [13].

Finally, the complexity comparisons among our and other three-layer non-Manhattan routing algorithms are shown in Table 4. It is seen that the time complexity of our approach is better than the other algorithms [11, 13].

\section{Conclusions}

We have described efficient bubble-sort-based algorithms for the two- and three-layer non-Manhattan channel routing problems. Based on the same routing model, the time complexities of our algorithm, two previous algorithms Chaudhary's [11], and Chen's [13] for the two-layer (and three-layer) non-Manhattan channel routings are $\mathrm{O}(k n)$, $\mathrm{O}\left(k n^{2}\right)$, and $\mathrm{O}\left(k^{2} n\right)$, respectively, where $k$ is the number 
of sorting passes required and $n$ is the number of twoterminal nets in a channel.

To further conduct the performance analysis of the three bubble-sort based algorithms, we tested them on a set of examples. Experimental results indicate that our algorithm requires only $1 \%$ more routing tracks than the optimal Chen's router [13] and the time improvement is over $40 \%$ of [13] on the average. Clearly, the time performance of our algorithm is better than previous algorithms [11, 13]. In future, we plan to integrate our bubble-sort router into an over-the-cell (OTC) channel router to reduce the final channel height in VLSI chip design.

\section{Acknowledgments}

The authors thank the anonymous referees for their constructive and helpful comments on this article. This work was supported by the National Science Council, Taipei, Taiwan, Republic of China, under grant NSC 862221-E-002-066.

\section{References}

1 HASHIMOTO, A., and STEVENS, J.: 'Wire routing by optimizing channel assignment within large apertures'. Proceedings of the 8th Design automation workshop, 1971, pp. 155-169

2 DEUTSCH, D.N.: 'A dogleg channel'. Proceedings of the 13th Design automation conference, 1976, pp. 425-433

3 YOSHIMURA, T, and KUH, E S . 'Efficient algorithms for channel routing', IEEE Trans. Comput.-Aided Des. Integr. Circuits Syst., 1982, 1, pp. $25-35$

4 RIVEST, R.L., and FIDUCCIA, C.M.: 'A greedy channel router'. Proceedings of the 19th Design automation conference, 1982, pp. 418-424

5 BURSTEIN, M., and PELAVIN, R.: 'Hierarchical channel router', VLSI J., 1983, 1, pp. 21-38

6 CONG, J., WONG, D.F., and LIU, C.L.: 'A new approach to three- and four-layer channel routing', IEEE Trans. Comput.-Aided Des. Integr. Circuits Syst., 1988, 7, pp. 1094-1104

7 SAIT, S.M., and YOUSSEF, H.: 'VLSI physical design automation' (McGraw-Hill, 1995), pp. 289-332

8 LODI, E., LUCCIO, F., and PAGLI, L.: 'A preliminary study of a diagonal channel-routing model', Algorithmica, 1989, 4, pp. 585-597

9 WANG, D.C.: 'Novel routing schemes for IC layout part I: two-layer channel routing'. Proceedings of the 28 th Design automation conference, 1991, pp. 49-53

10 WANG, D.C., and KUH, E.S.: 'New algorithms for two- and three-layer channel routing', Int. J. Circuit Theory Appl., 1991, 19, pp. 525-549

11 CHAUDHARY, K., and ROBINSON, P.: 'Channel routing by sorting', IEEE Trans. Comput.-Aided Des. Integr. Circuits Syst., 1991, 10, pp. $754-760$

12 KNUTH, D.E.: 'Sorting and searching, 3' (Addison-Wesley, Reading, MA, 1973)

13 CHEN, C.Y.R., HOU, C.Y., and SINGH, U.: 'Optimal algorithms for bubble sort based non-Manhattan channel routing', IEEE Trans. Comput.-Aided Des. Integr. Circuits Syst., 1994, 13, pp. 603-609 